Canad. Math. Bull. Vol. 23 (4), 1980

\title{
INFINITESIMAL ISOMETRIES ON COMPACT MANIFOLDS
}

\author{
BY \\ CHAO-CHU LIANG*
}

Let $X$ denote a non-vanishing infinitesimal isometry on a compact Riemannian manifold $M^{n}$. Let $A(M)=\left\{A^{k}(M), d\right\}_{0 \leq k \leq n}$ denote the deRham complex of $M$. We write $i(X)$ for the operator of interior product, and $L(X)$ the Lie derivative on the elements of $A(M)$. We define $E(M)=$ $\{u \in A(M) \mid i(X) u=0, L(X) u=0\}$. The cohomology $H^{i}(E(M))$ of the complex $\{E(M), d\}$ is of finite type, since the deRham cohomology $H^{i}(M)$ is, [5, p. 189]. We define the Euler characteristic of the "orbit space" $M / X$ by $\chi(M / X)=$ $\sum_{i=0}^{n-1}(-1)^{i} H^{i}(E(M))$.

The purpose of this paper is to show that when $\chi(M / X) \neq 0$, then $X$ has at least one periodic orbit; and $\chi(M / X)$ is equal to the Euler number (in the singular cohomology) of some compact space.

From now on, we assume that all the manifolds are compact and without boundaries, unless stated otherwise.

1. Let $X$ be a non-vanishing infinitesimal isometry on a compact Riemannian manifold $\boldsymbol{M}^{n}$ with metric $\langle$,$\rangle . The following proposition was proved in [8]:$

Proposrtion 1. If there exists a non-vanishing vector field $Y$ on $M$ such that $\{X, Y\}$ forms a set of linear independent commuting (i.e., $L(X) Y=[X, Y]=0)$ vector fields on $M$, and $\langle X, Y\rangle=0$. Then $\chi(M / X)=0$.

Since $M$ is compact, the group of isometries $I(M)$ is compact, and the 1-parameter group $\left\{g_{t}\right\}$ generated by $X$ is densely embedded in a torus group $T^{k} \subseteq I(M)$. Let $\mathscr{L}\left(T^{k}\right)=R^{k}$ denote the Lie algebra of $T^{k}$. Using the notation in [5, p. 188], we see that $X=Z_{h}$, the vector field on $M$ induced by some $h \in \mathscr{L}\left(T^{k}\right)$ and the $T^{k}$-action.

THeOrem 1. Let $X$ be a non-vanishing infinitesimal isometry on a compact Riemannian manifold $M$. If $\chi(M / X) \neq 0$, then $X$ has a periodic orbit.

Proof. By abuse of language, we also use $\langle$,$\rangle to denote the metric on T^{k}$. Recall that $X=Z_{h}$. We define an affine subspace $A$ of $\mathscr{L}\left(T^{k}\right)=R^{k}$ by

$$
A=\left\{\beta \in \mathscr{L}\left(T^{k}\right) \mid\langle\beta, h\rangle=0\right\} .
$$

\footnotetext{
* Supported by the University of Kansas General Research Fund.

Received by the editors February 20, 1979.
} 
If $X$ has no periodic orbit, then the dimension of every $T^{k}$-orbit is greater than 1. Therefore the dimensions of all isotropy subgroups are less than $k-1$. Because $T^{k}$ is abelian, two subgroups $S_{1}$ and $S_{2}$ are conjugate if and only if they are the same. It then follows from the theorem on the finiteness of number of orbit types $[1$, p. 93$]$ that the set $B$ of isotropy subgroups is finite. We write $B=\left\{S_{1}, \ldots, S_{r}\right\}$. Let $A_{j} \subseteq \mathscr{L}\left(T^{k}\right)$ be the subspace corresponding to $S_{j}$. Since $\operatorname{dim} S_{j} \leq n-2$, there exists an element $\beta \in A-\bigcup_{j} A_{j}$. The vector field $Y$ on $M$ defined by $Y=Z_{\beta}$ satisfies the assumption of Proposition 1 , hence $\chi(M / X)=0$. q.e.d.

2. For a subgroup $H$ of $T^{k}$, we let $F(H)$ denote the fixed point set of $H$ under the $T^{k}$-action on $M$. The set of periodic orbits of a non-vanishing infinitesimal isometry $X$ on $M$ is the finite union $F\left(H_{1}\right) \cup \cdots \cup F\left(H_{r}\right)$, where each $H_{\mathrm{j}}$ is a codimension 1 subtorus of $T^{k}$. Each $F_{\mathrm{j}}=F\left(H_{\mathrm{j}}\right)$ is a closed submanifold of $M$. The circle group $S^{1}=T^{k} / H_{j}$ acts on $F_{j}$ without fixed points, and the orbits of the $S^{1}$-action are the same as the orbits of $X$. We write $H_{\text {sing }}^{i}(W)$ for the $i$ th singular cohomology of a space $W$. We define $\chi_{\text {sing }}\left(F_{j} / S^{1}\right)=\sum_{i}(-1)^{i} H_{\text {sing }}^{i}\left(F_{j} / S^{1}\right)$.

TheOREM 2. $\chi(M / X)=\sum_{\mathrm{j}} \chi_{\text {sing }}\left(F_{\mathrm{j}} / S^{1}\right)$.

Proof. For each $F_{j}$ (which may have several components), we may choose a closed $T^{k}$-invariant tubular neighborhood $N_{j}$ in $M,[2$, p. 306]. We may further assume that $N_{i} \cap N_{j}=\emptyset$ for $i \neq j$, and $N=M-\bigcup_{j} N_{j}$ is a manifold with boundary. Since the boundaries $\partial N_{j}$ 's are $T^{k}$-invariant, we may still define $E\left(N_{j}\right)$ as in [5, pp. 188-189]. A Mayer-Vietoris sequence can be proved for this case in the usual fashion.

From Theorem 1 above, we see that $\chi(N)=0$, and $\chi\left(\partial N_{j}\right)=0$ for all $j$. It then follows from the Mayer-Vietoris sequence that

$$
\chi(M / X)=\sum_{j} \chi\left(N_{j} / X\right)
$$

Since $N_{j}$ is a $T^{k}$-invariant (hence $g_{t}$-invariant, where $g_{t}$ is the 1-parameter group generated by $X$ ) tubular neighborhood of $F_{i}$, there exists a $g_{t}$-equivariant retraction from $N_{j}$ to $F_{j}$. This induces a chain equivalence between $E\left(N_{j}\right)$ and $E\left(F_{j}\right)$, hence an isomorphism between $H^{i}\left(E\left(N_{j}\right)\right)$ and $H^{i}\left(E\left(F_{j}\right)\right)$. Thus, we have

$$
\chi\left(N_{\mathrm{j}} / X\right)=\chi\left(F_{\mathrm{j}} / X\right)=\chi\left(F_{\mathrm{j}} / S^{1}\right) .
$$

By using the same argument in the sheaf-theoretic proof of the deRham theorem, it can be shown that $H^{i}\left(E\left(F_{j} / S^{1}\right)\right)$ is the same as the Cech cohomology $\check{H}^{i}\left(F_{j} / S^{1}\right)$, [5, p. 192].

Since $S^{1}$ acts on $F_{j}$ with finite isotropy subgroups, the orbit space $F_{j} / S^{1}$ is a 
$V$-manifold, a space locally homeomorphic to the orbit space $R^{b} / K$ of an orthogonal action on the Euclidean space $R^{b}$ by a finite group $K$, [7]. According to [6], $R^{b} / K$ can be triangulated, hence it is an Euclidean neighborhood retract (ENR). So the compact space $F_{j} / S^{1}$, as a union of finitely many $R^{b} / K$ 's, is also an ENR, [4, p. 82]. But for ENR's, Čech-cohomology coincides with singular cohomology, [4, p. 285]. Therefore, we have

$$
\chi(M / X)=\sum_{j} \chi_{\text {sing }}\left(F_{j} / S^{1}\right) . \quad \text { q.e.d. }
$$

3. For a non-vanishing infinitesimal isometry $X$ on the $(2 n+1)$-dimensional sphere $S^{2 n+1}$, it can be checked that $\chi\left(X^{2 n+1} / X\right)=n+1$, [5, pp. 188-189], [8]. Therefore, there doesn't exist a vector field $Y$ on $S^{2 n+1}$ satisfying the conditions stated in Proposition 1. Here we prove a more general result.

THEOREM 3. Let $M^{n}$ denote a compact Riemannian manifold having the integral cohomology of an $n$-sphere $S^{n}$, and let $X$ be a non-vanishing infinitesimal isometry on $M$. If $Y$ is a vector field on $M$ prevserving the orbits of $X$, then $\{X, Y\}$ must be linearly dependent at some point of $M$.

Proof. Suppose there exists a non-vanishing vector field $Y$ such that $X$ and $Y$ are linearly independent, and $Y$ preserves the orbits of $X$ (i.e. the Lie bracket $L_{Y} X=[Y, X]=f X$, for a function $f$ on $\left.M\right)$. As before, there exists a $T^{k}$-action on $S^{n}$ induced by $X$.

Let $\mathrm{H}$ denote a codimension 1 torus subgroup of $T^{k}$, and $n(H)$ denote the dimension of the fixed point set $F(H)$. Then we have the following Borel formula [1, p. 175]:

$$
n+1=\sum_{H}(n(H)+1)
$$

where $H$ runs through the torus subgroup of codimension 1 . Therefore, there exists a collection of codimension 1 tori $H_{1}, \ldots, H_{c}$ with nonempty fixed point set $F\left(H_{j}\right)=F_{j}$. Let $n_{j}=n\left(H_{j}\right)$. We also know that $F_{j}$ is an integral cohomology $n_{j}$-sphere, and $n-n_{j}$ is even [1, p. 175].

As in section 2 , the circle group $S^{1}=T^{k} / H_{j}$ acts on the submanifold $F_{j}$ without fixed point, and the orbits of the $S^{1}$-action coincide with the orbits of $X$. The orbit space $F_{j} / S^{1}$ is a $V$-manifold. Since $Y$ preserves the orbits of $X$, it induces a non-vanishing vector field $W_{j}$ on the orbit space $F_{j} / S^{1}$. This implies that $\chi\left(F_{j} / S^{1}\right)=0$, [7]. But according to [3], $F_{j} / S^{1}$ has the integral cohomology of a complex projective space, hence

$$
\chi\left(F_{j} / S^{1}\right)=\frac{n_{j}+1}{2}
$$

a contradiction. q.e.d. 


\section{REFERENCES}

1. A. Borel, Seminar on transformation groups. Ann. of Math. Studies \#46. Princeton Univ. Press 1960.

2. G. Bredon, Introduction to compact transformation groups. Academic Press, New York 1972.

3. P. E. Connor and E. E. Floyd, Orbit spaces of circle groups of transformation. Ann. of Math. 67 (1958), 90-98.

4. A. Dold, Lectures on algebraic topology. Springer-Verlag, New York-Heidelberg-Berlin 1972.

5. W. Greub, S. Halperin, and R. Vanstone, Connections, curvature, and cohomology. Vol. II, Academic Press, New York-London 1973.

6. S. Illman, Smooth equivariant triangulation of $G$-manifolds for $G$ a finite group. Math. Ann. 233 (1978), 199-220.

7. C. C. Liang, Vector fields on $V$-manifolds, and locally free $G \times R^{1}$-actions on manifolds. Indiana Univ. Math. J. 27 (1978), 349-352.

8. C. C. Liang, Vector fields orthogonal to a non-vanishing infinitesimal isometry. Proc. Amer. Math. Soc. 75 (1979), 145-148.

DEPARTMENT OF MATHEMATICS

UNIVERSITY OF KANSAS

LAWRENCE, KANSAS 66045. 